\title{
Hematoma Intramural da Artéria Pulmonar Associado a Dissecção Aórtica Aguda
}

\section{Pulmonary Arterial Intramural Hematoma Due to Acute Aortic Dissection}

Lucas de Pádua Gomes de Farias, ${ }^{1}$ Ana Cristina Favaretto, ${ }^{1}$ Luciana de Pádua Silva Baptista, ${ }^{1}$ Gustavo Borges da Silva Teles ${ }^{\top}$

UnitedHealth Group Brazil, ${ }^{1}$ São Paulo, SP - Brasil

Paciente do sexo masculino, 54 anos, tabagista, é admitido na unidade de emergência com dor torácica aguda e dispneia. Procedeu-se a investigação por meio da angiotomografia computadorizada de tórax que evidenciou extensa dissecção da aorta torácica com início no segmento ascendente (tipo A de Stanford) associada a hematoma intramural do tronco da artéria pulmonar e dos seus ramos principais, mais evidente à direita, que determina redução luminal pulmonar local, além de um pequeno hematoma mediastinal para-aórtico e subaórtico. Não havia sinais de tromboembolismo pulmonar e a avaliação do parênquima não evidenciou sinais de hemorragia pulmonar (Figuras 1 e 2).

A dissecção aórtica aguda é uma condição de alto risco de vida e o hematoma mediastinal que disseca a bainha das artérias pulmonares é considerado uma rara complicação ${ }^{1-3}$ que pode simular tromboembolismo pulmonar e vasculites. ${ }^{4}$ Isso geralmente ocorre porque, ao nível logo acima da válvula aórtica, a aorta ascendente e o tronco da artéria pulmonar compartilham uma adventícia comum, que se torna o pericárdio visceral caudalmente. ${ }^{1,4,5} \mathrm{Na}$ maioria dos casos, há a ruptura da cama média adjacente à artéria pulmonar direita, e o sangue flui da aorta ascendente para o espaço intersticial que limita as artérias pulmonares (hematoma intramural) (Figura 3), podendo se estender aos septos interlobulares ou mesmo aos alvéolos por meio do interstício peribroncovascular. ${ }^{1,2,4}$ Alguns casos isolados de hematoma da artéria pulmonar podem estar relacionados à patência do ducto arterioso, hipertensão pulmonar e desordens do tecido conjuntivo. ${ }^{6-9}$
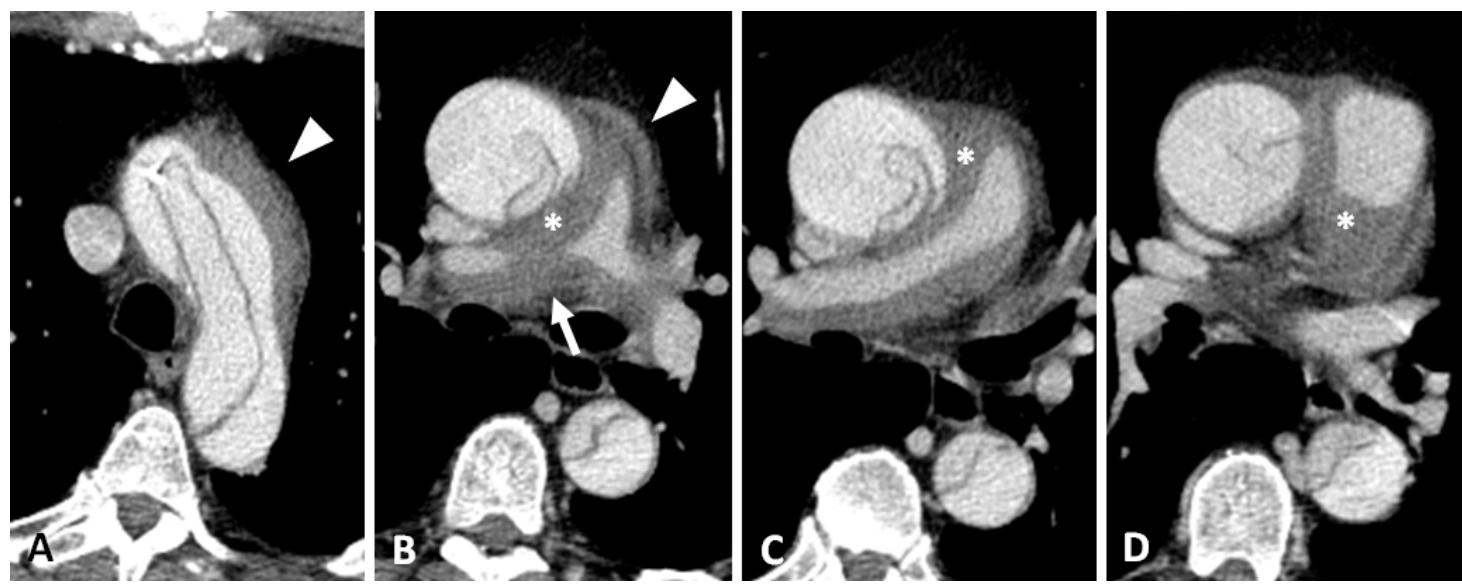

Figura 1 - Angiotomografia computadorizada de tórax, em aparelho com 16 fileiras de detectores ( $A$ a $D$ - reconstrução multiplanar axial), evidencia dissecção aórtica aguda (tipo A de Stanford) associada a hematoma secundário (asterisco), envolvendo o tronco da artéria pulmonar e seus ramos principais, mais evidente à direita, determinando redução luminal da sua porção proximal (seta branca). Note também o hematoma mediastinal nas regiões para-aórtica e subaórtica (cabeça de seta branca).

\section{Palavras-chave}

Dor no Peito; Hematoma; Artéria Pulmonar; Angiografia por Tomografia Computadorizada/métodos; Artéria Pulmonar; Aneurisma Dissecante.

Correspondência: Lucas de Pádua Gomes de Farias •

R. Conselheiro Brotero, 1486 - Higienópolis. CEP 01232-010, São Paulo, $\mathrm{SP}$ - Brasil

E-mail: lucasdpadua@hotmail.com

Artigo recebido em 14/07/2020, revisado em 21/10/2020, aceito em 02/12/2020

DOI: https://doi.org/10.36660/abc.20200775 


\section{Imagem}
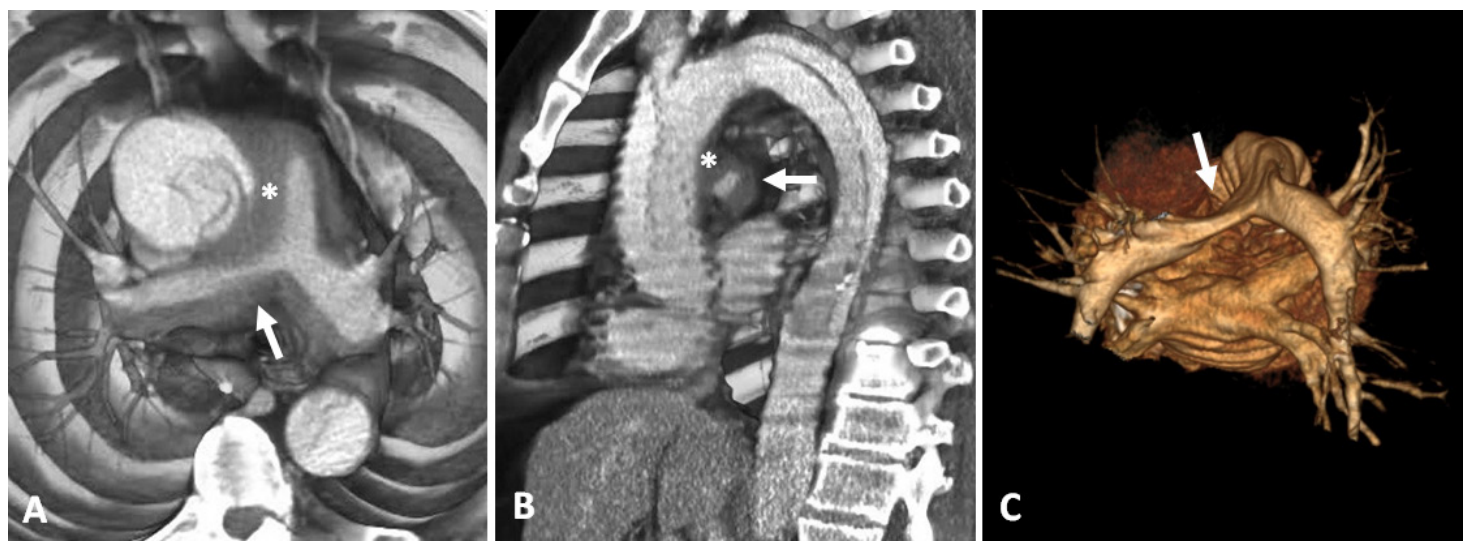

Figura 2 - Reconstruções tridimensionais de angiotomografia computadorizada de tórax, em aparelho com 16 fileiras de detectores, evidenciam dissecção aórtica aguda (tipo A de Stanford) associada a hematoma secundário (asterisco) envolvendo o tronco da artéria pulmonar e seus ramos principais, mais evidente à direita, determinando redução luminal da sua porção proximal (seta branca).

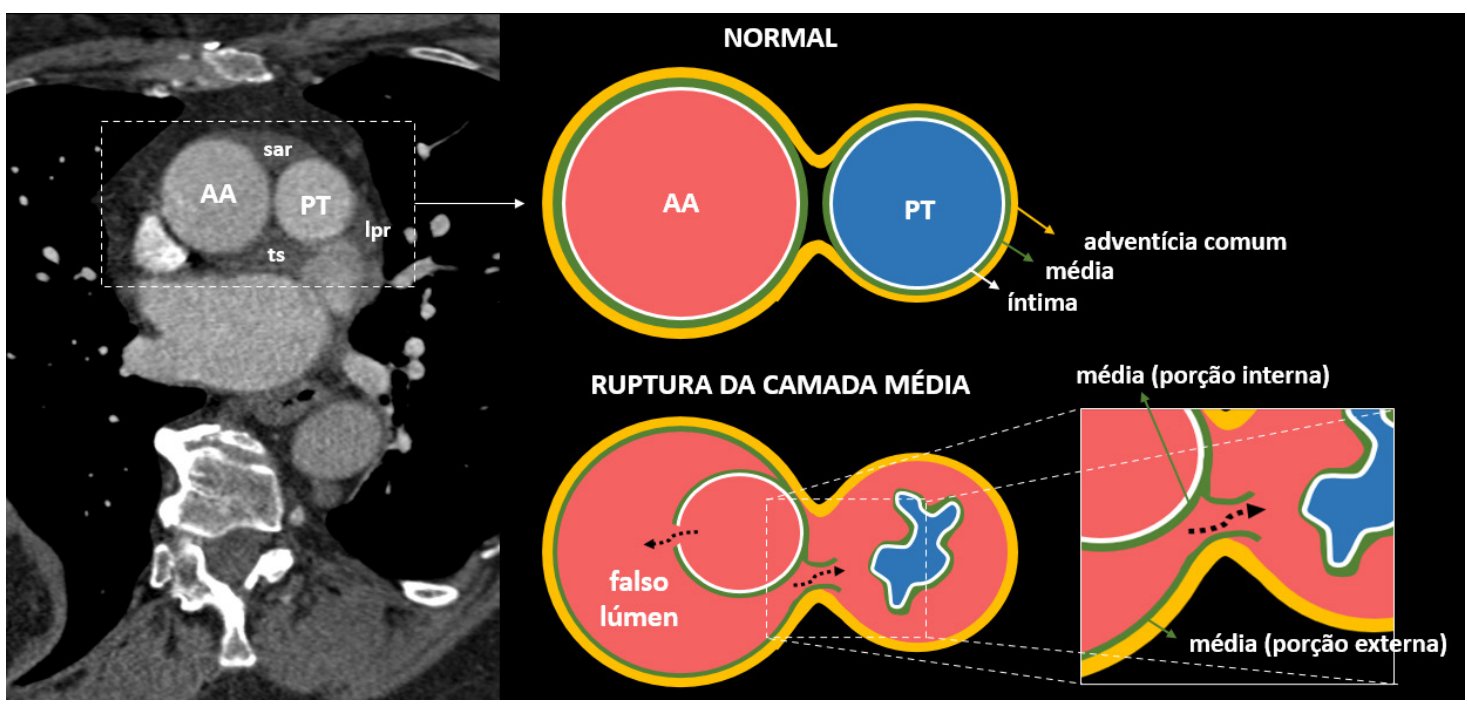

Figura 3 - Desenho esquemático evidencia a ruptura da porção mais externa da camada média no falso lúmen da dissecção aórtica, adjacente à artéria pulmonar, resultando extravasamento de sangue na adventícia comum entre a aorta e a artéria pulmonar que pode estreitar o lúmen arterial pulmonar. AA: aorta ascendente; Ipr: recesso pulmonar esquerdo do seio transverso; PT: tronco pulmonar; sar: recesso aórtico superior; ts: seio transverso. Adaptado de Roberts. ${ }^{5}$

\section{Contribuição dos autores}

Concepção e desenho da pesquisa, Obtenção de dados, Análise e interpretação dos dados e Redação do manuscrito: Farias LPG, Favaretto AC; Revisão crítica do manuscrito quanto ao conteúdo intelectual importante: Baptista LPS; Teles GBS.

Potencial conflito de interesse

Não há conflito com o presente artigo

\section{Fontes de financiamento}

O presente estudo não teve fontes de financiamento externas.

\section{Vinculação acadêmica}

Não há vinculação deste estudo a programas de pós-graduação.

\section{Aprovação ética e consentimento informado}

Este artigo não contém estudos com humanos ou animais realizados por nenhum dos autores. 


\section{Referências}

1. Sueyoshi E, Matsuoka Y, Sakamoto I, Uetani M. CT and clinical features of hemorrhage extending along the pulmonary artery due to ruptured aortic dissection. Eur Radiol. 2009;19(5):1166-74.

2. Castañer E, Andreu M, Gallardo X, Mata JM, Cabezuelo MA, Pallardo Y,et al. CT in Nontraumatic Acute Thoracic Aortic Disease: Typical and Atypical Features and Complications. RadioGraphics. 2003; 23(Suppl1):S93-110.

3. Buja LM, Ali N, Fletcher RD, Roberts WC. Stenosis of the right pulmonary artery: a complication of acute dissecting aneurysm of the ascending aorta. Am Heart J. 1972; 83:89e92

4. Nasrallah A, Goussous Y, El-Said G, Garcia E, Hall RJ. Pulmonary artery compression due to acute dissecting aortic aneurysm: clinical and angiographic diagnosis. Chest. 1975;67(2):228-30.
5. Roberts WC. Aortic dissection: Anatomy, consequences, and causes. Am Heart J. 1981; $101: 195-214$

6. Kang EJ, Lee KN, Kim I, Lee J. Spontaneously developed pulmonary arterial intramural hematoma that mimicked thromboembolism. Korean J Radiol. 2012;13:496e499.

7. Steurer J, Jenni R, Medici TC, Kolrath TH. Dissecting aneurysm of the pulmonary artery with pulmonary hypertension. Am Rev Respir Dis. 1990;142:1219-21.

8. Rosenson RS, Sutton MS. Dissecting aneurysm of the pulmonary trunk in mitral stenosis. Am J Cardiol. 1986; 58(11):1140-1.

9. Mohammad K, Sahlol M, Egiebor O, Sadikot RT. Idiopathic pulmonary artery dissection: a case report. J Med Case Rep. 2009; 3(1):7426. 